\title{
Medición del mejoramiento de la lectura comprensiva del estudian- tado de primer ingreso de la UNAH mediante la asignatura de Español General (año 2017)
}

Measurement of the improvement of the comprehensive reading of the first-year students of the UNAH through the subject of General Spanish (year 2017)

DOI 10.5377pc.v0i16.8100

\author{
Waldina Mejía ${ }^{1}$ \\ Socorro Castellón ${ }^{2}$ \\ Ramón Enamorado ${ }^{3}$
}

\section{RESUMEN}

Debe evitarse la frecuente práctica de decidir reformas o cambios educativos sin evaluaciones objetivas de lo que se pretende corregir. Con esta investigación descriptiva se midió a través de un diseño pretest-postest la mejora de la lectura comprensiva de los y las estudiantes de primer ingreso después de cursar la asignatura de Español General (EG-011) en el primer período 2017 en la CU-UNAH, a fin de obtener datos concretos para reformar el programa de esa asignatura en función del logro de esa destreza mental. También como una forma de evaluar el logro de la universidad como institución. Se eligió la lectura comprensiva por ser base para las demás destrezas mentales académicas y ser relativamente más fácil de evaluar. Los datos se procesaron con los programas EPI-INFO y SPSS y la mejora su calculó con diferencia de promedios. Los resultados indican que ésta es muy pequeña $(4.23 \%)$ y por tanto hay que reformar el programa. Además del instrumento, se comenzó a indagar sobre prácticas didácticas mejores y se aplicó una encuesta sobre 14 factores socioeconómicos para saber su relación con el logro de esta destreza y así guiarse en esta reforma. Es necesario dar seguimiento a esta investigación para confirmar las tendencias obtenidas.

Palabras clave: Mejora de lectura comprensiva, didáctica del español, psicolingüística de la lectura, autoevaluación universitaria

\footnotetext{
${ }^{1}$ Beneficiaria de una beca básica. Máster en Literatura Centroamericana. Departamento de Letras, Facultad de Humanidades y Artes, UNAH: waldina.mejia@unah.edu.hn, waldinapoesia@gmail.com

${ }^{2}$ Asesora, PhD, teoría y aplicación de los instrumentos, UNAH: scastellon@gmail.com

${ }^{3}$ Asesor, Máster, apoyo en programación y manejo estadístico, UNAH: renamoradopineda@yahoo.com
} 


\section{ABSTRACT}

The frequent practice of deciding reforms or educational changes without objective evaluations of what is intended to be corrected should be avoided. With this descriptive research, the improvement of the comprehension reading of the first-year students after studying the subject of General Spanish (EG-011) in the first period 2017 in the $\mathrm{CU}$ - was measured through a pretest-posttest design. UNAH, in order to obtain concrete data to reform the program of that subject based on the achievement of that mental skill. Also as a way to evaluate the achievement of the university as an institution. Comprehensive reading was chosen as the basis for other academic mental skills and relatively easier to assess. The data was processed with the EPI-INFO and SPSS programs and the improvement was calculated with a difference of averages. The results indicate that this is very small (4.23\%) and therefore the program must be reformed. In addition to the instrument, they began to investigate better teaching practices and a survey was applied on 14 socioeconomic factors to know their relationship with the achievement of this skill and thus be guided in this reform. It is necessary to follow up on this research to confirm the trends obtained.

Keywords: C omprehensive reading improvement, spanish didactics, reading psycholinguistics, university self-assessment. 


\section{INTRODUCCIÓN}

Este artículo quiere llamar la atención sobre prácticas de tomar decisiones de reforma o cambios educativos basadas en opiniones u observaciones empíricas o en "modas" de otros lugares; prácticas que no deben seguir. Esta investigación probó que se puede contar con evaluaciones objetivas como insumos para decidir reformas 0 cambios educativos programáticos y/o políticos, al concretar una forma objetiva y factible de conocer la efectividad de la asignatura de Español General (y de paso, de esta universidad como institución educativa) para elevar el nivel de la destreza mental de lectura comprensiva de los recién ingresados a la UNAH.

\section{Elementos teóricos usados}

Para efectos de una más ágil comunicación, el instrumento desarrollado y aplicado en esta investigación fue bautizado como "Prueba de lectura Comprensiva" (PLEC), en homología con la Prueba de Aptitud Académica (PAA) y los test PISA. Se eligió la destreza de lectura comprensiva por ser base para las demás destrezas mentales académicas y ser relativamente más fácil de evaluar. Se usaron varias referencias especializadas. La obligada para este tipo de evaluación es el programa PISA, del cual se obtuvo algunas nociones teóricas, como la discriminación entre texto continuo (o en prosa o verso) y texto discontinuo (cuadros, diagramas, esquemas, imágenes, gráficos, etc) y la clasificación por su uso en público, educativo, literario, informativo, expositivo, personal y argumentativo o polémico. En este estudio no se usó el texto de tipo instruccional por limitación de tiempo.

Para este estudio se establecieron cuatro niveles de lectura: estructural, primer nivel, segundo nivel y global. La lectura estructural es la que permite el reconocimiento de las partes del texto, en la que intervienen los signos de puntuación y los espacios del texto. La lectura de primer nivel o lineal, es la de comprensión literal de frases y oraciones aisladas. La lectura comprensiva de segundo nivel o de intra e interpárrafo, es la que permite la identificación y comprensión de la idea principal del párrafo y de sus relaciones con las de los demás párrafos. La lectura global es la de identificación y comprensión del tema, intención y mensaje principal del texto. Como uno de los aportes de este trabajo, los ítems evalúan estos distintos niveles de lectura en cada reactivo textual de distinto tipo. Para evaluar lectura global también se usó el "test Cloze", que no lo incluye PISA. Éste test consiste en un texto al cual se le han quitado ciertas palabras, las cuales deben ser restituidas por el examinado, escogiéndolas de una lista dada, en esta versión. 


\section{Dificultades}

Inicialmente se pensó en usar los resultados del área verbal de la Prueba de Aptitud Académica (PAA) como pretest y volver a aplicar la misma como postest; pero la PAA es propiedad de College Board, en Puerto Rico, que la UNAH contrata para este trabajo. College Board cobra $\$ 11$ por cada prueba aplicada (que debe enviárseles para su revisión). Con la beca de la DICYP no podían cubrirse costos tan altos. Por tanto, hubo que desarrollar un instrumento de evaluación ad hoc, el PLEC, que debe evaluar lo que pretende y cuyos reactivos textuales deben adaptarse a la situación y dialecto particular de donde se aplicarían. Para asegurarse de cumplir estos dos requisitos se realizó su validación o pilotaje, de la cual se hablará posteriormente.

Por la prolongada huelga de estudiantes de año 2016, casi todo el trabajo de la primera etapa se acumuló en septiembre. Por esto, en noviembre 22, la DICYP aprobó realizar la segunda etapa en el primer período 2017. Otra dificultad fue que a inicios del 2017 se ofertó muchas secciones de Español General que se impartirían en el edificio C-3, pero la UNAH lo cerró para repararlo y remodelarlo. Esto generó varias dificultades, mientras se les hallaba espacio a las muchas secciones que se quedaron sin aula. Entre otras, atrasó el obtener en el tiempo programado la información requerida por esta investigación sobre matrícula y secciones existentes entonces. Hasta el jueves de la primera semana de febrero se obtuvo esta información, lo que provocó que hubo que trabajar dos días y dos noches seguidos para lograr seleccionar las secciones más adecuadas y obtener el permiso de los respectivos docentes; hubo casos en que se debió cambiar la sección elegida al no aceptar el docente participar en el estudio (la principal razón es que se requería dos días de pretest y dos de postest, 4 días menos para las clases normales). También se debió posponer una semana la aplicación del pretest, con el debido permiso de la DICYP.

Por el hecho de que todas las secciones de EG-011 no contaban desde el inicio con su respectiva aula, se supuso que habría mucha deserción e irresponsabilidad de parte de los estudiantes y que se perdería mucha información. Por eso, se aplicaron muchos más instrumentos por sección de los proyectados. Además se agregaron dos secciones más (total 17 secciones). Gracias a esta medida, se logró sostener la cantidad mínima de la muestra estadística exigida porque, en efecto, varios alumnos no se presentaron al segundo día de aplicación del pretest y el postest; 0 a todo el postest.

Aparte, algunos resultados del nivel de lectura estructural salieron incoherentes (casi todos negativos en la diferencia de promedios, mientras todos los demás tipos de test -más difíciles- salieron positivos). Hubo que revisar todo el proceso y se encontró que 
la pauta tenía dos errores. Se corrigió y se corrió de nuevo toda la programación estadística.

\section{Tipo y alcances de la Investigación}

Para efectos de una más ágil comunicación, los instrumentos desarrollados y aplicados en esta investigación serán llamados "Prueba de lectura Comprensiva" (PLEC), en homología con la Prueba de Aptitud Académica (PAA) y los test PISA. Esta investigación solo se hizo en la ciudad universitaria de la UNAH en Tegucigalpa; es cuantitativa, descriptiva y novedosa en el país. Este alcance es el adecuado porque no hay estudios previos en Honduras y en el UNAH sobre el tema, hubo que comenzar de cero: La exploración se hizo directamente a través de los expertos, docentes de Español General de la UNAH, en el 2015. Los hallazgos dieron ideas o tendencias para nuevas investigaciones relativas al tema.

El diseño. De tipo PRETEST-POSTEST, pues en estos cursos es muy difícil contar con un(os) grupo(s) control; el pretest funciona como "control" para conocer, por comparación con los resultados del postest, si hay cambios y mejora en el nivel de lectura comprensiva de los sujetos de la investigación.

Validación del Instrumento. Se desarrolló y pilotó el instrumento de evaluación el tercer período 2016. Para la validez de contenido y de constructo, el instrumento fue revisado por un grupo de docentes de especialistas del Departamento de Letras que imparten la asignatura de Español General (EG-011). A groso modo, ellos hicieron algunas correcciones de forma (errores en mayúsculas, tildes y puntuaciones). También, el texto polémico Grafiti, se dejaron solo dos opiniones distintas y no tres, puesto que APP, solo incluye dos y debe tratar de mantenerse el mismo grado de dificultad (sugerencia del Lic. Carlos Velázquez). La validación de constructo se hizo también con la revisión de Socorro Castejón, doctora en Evaluación Educativa, y Ramón Enamorado, del Departamento de Matemáticas, quien prestó sus servicios profesionales como estadígrafo. Éste le dio el formato final a la encuesta y propuso realizar una prueba de diferencia de promedios entre los resultados del prestest y postest, además de obtener el índice de Pearson, el Cronbach y los otros procedimientos estadísticos.

La digitalización de los datos del pilotaje y el tratamiento estadístico de los mismos, se atrasó porque los horarios libres del laboratorio de cómputo de Letras no coincidían con las horas libres de clases de los estudiantes digitadores. Éstos tuvieron que trabajar en su casa con su propio equipo. Por eso se pidió cambio de presupuesto 
para comprar una computadora portátil para digitalizar y otros trabajos, que se usó debidamente. Se revisó todas las digitalizaciones y hubo que repetir varias. Por fortuna, había suficiente lapso del tiempo antes del postest.

\section{Aplicación de la PLEC}

El instrumento ya validado, se aplicó al inicio y a final del primer período académico 2017 (14 y 15 de febrero; 19 y 20 de abril), junto con una encuesta de factores socioeconómicos, con cuyos datos se relacionaron los resultados de la medición.

Muestra. Un total de 3980 de estudiantes fue la población de primer ingreso matriculados en la asignatura de Español General en las distintas secciones de lunes a jueves desde las 06.00 a las 20.00 horas en el primer período 2017. Solo se consideró los cursos presenciales de lunes a jueves porque los otros horarios y modalidades tienen otra dinámica, que sería un fuerte sesgo en la investigación. El listado de las secciones, docentes asignados y la matrícula se obtuvieron de la Jefatura de la Escuela de Letras y se confirmaron con un censo en las aulas. La muestra estadística al $95 \%$ de confianza y $5 \%$ de error es 350 . Se distribuyó esta cantidad entre los conglomerados naturales que existen (las secciones, una por hora, desde las $6.00 \mathrm{a}$ las $20.00 \mathrm{H}$ ). Logramos 4341 informantes válidos, lo que equivale a 97.26 de confianza y 4.44 de error ${ }^{4}$.

Las secciones participantes ${ }^{5}$ fueron 15 (ver Tabla N. 1). Para seguridad de mantener la muestra requerida, se aplicaron muchos más instrumentos por sección de los proyectados. Además se agregaron dos secciones: 1501, y 1901 (total 17 secciones). Ésta última se eliminó en el postest.

Como se ve en la tabla N 1, en las secciones de las siete, ocho y once a.m. se perdió mayor información. Por eso, de las dos secciones extras, se compensó con la sección 1501, que aún predomina en población joven que no trabaja (y se eliminó la 1901, cuyo mayor grupo es de más edad y trabaja).Se digitalizó esta información y se sometió al tratamiento estadístico con los programas EPI-INFO Y SPSS. Los procedimientos usados son los normales en estos casos y se verán adelante.

\footnotetext{
${ }^{4}$ Se usó la calculadora de muestras de http://med.unne.edu.ar/biblioteca/calculos/calculadora.htm).

${ }^{5}$ Había horarios en que solo había docentes jóvenes; en estos casos se eligió a quien más años de trabajo tiene en la UNAH. Requirió muchísimo trabajo realizar esta selección, en lo posible conjugando todos los criterios establecidos (se priorizaban: más años de experiencia docente en la UNAH, mejor titulación, empleado permanente).
} 
Tabla 1.

\begin{tabular}{|c|c|c|c|c|c|c|c|c|}
\hline & \multicolumn{4}{|c|}{ PRETEST } & \multicolumn{4}{|c|}{ POSTEST } \\
\hline 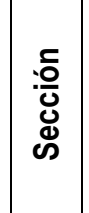 & 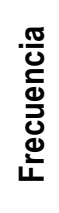 & $\alpha^{\circ}$ & $\begin{array}{l}\frac{0}{\frac{0}{0}} \\
\frac{\pi}{20} \\
\frac{0}{0}\end{array}$ & 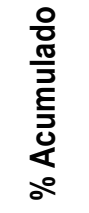 & 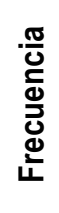 & ○̊ & $\begin{array}{l}\text { 은 } \\
\frac{\mathrm{T}}{\mathrm{N}} \\
\frac{2}{20}\end{array}$ & 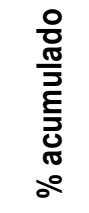 \\
\hline 600 & 42 & 5.8 & 5.8 & 5.8 & 26 & 5.9 & 5.9 & 5.9 \\
\hline 0701 & 38 & 5.3 & 5.3 & .1 & 15 & 3.4 & 3.4 & 9.4 \\
\hline 0803 & 24 & 3.3 & 3.3 & $t .5$ & 12 & 2.7 & 2.7 & 12.1 \\
\hline 9903 & 52 & 7.2 & 7.2 & 21.7 & 37 & 8.4 & 8.4 & 20.5 \\
\hline 1001 & 50 & 7.0 & 7.0 & 28.7 & 31 & 7.1 & 7.1 & 27.6 \\
\hline 1102 & 40 & 5.6 & 5.6 & 34.2 & 15 & 3.4 & 3.4 & 31.1 \\
\hline 1204 & 46 & 6.4 & 6.4 & 40.6 & 26 & 5.9 & 5.9 & 37.0 \\
\hline 1301 & 35 & 4.9 & 4.9 & 45.5 & 27 & 6.2 & 6.2 & 43.2 \\
\hline 401 & 49 & 6.8 & 6.8 & 52.3 & 25 & 5.7 & 5.7 & 48.9 \\
\hline 501 & 44 & 6.1 & 6.1 & 58.4 & 37 & 8.4 & 8.4 & 57.3 \\
\hline 1502 & 47 & 6.5 & 6.5 & 65.0 & 34 & 7.8 & 7.8 & 65.1 \\
\hline 1600 & 51 & 7.1 & 7.1 & 72.0 & 35 & 8.0 & 8.0 & 73.1 \\
\hline 1701 & 42 & 5.8 & 5.8 & 77.9 & 26 & 5.9 & 5.9 & 79.0 \\
\hline 1800 & 34 & 4.7 & 4.7 & 82.6 & 26 & 5.9 & 5.9 & 84.9 \\
\hline 1900 & 38 & 5.3 & 5.3 & 87.9 & 31 & 7.1 & 7.1 & 92.0 \\
\hline 1901 & 40 & 5.6 & 5.6 & 93.5 & & & & \\
\hline 2000 & 47 & 6.5 & 6.5 & 100.0 & 35 & 8.0 & 8.0 & 100.0 \\
\hline Total & 719 & 100.0 & 100.0 & & 438 & 100.0 & 100.0 & \\
\hline
\end{tabular}

\section{Análisis e interpretación de resultados de la investigación por objetivos}

El análisis se desarrollará siguiendo los objetivos de esta investigación.

Antes, se resumen los datos más importantes de la información recabada. Ambas muestras tienen las siguientes estadísticas (Ver tabla 2):

\section{Tabla 2. Estadísticos de la escala PRETEST Y POSTEST}

\begin{tabular}{|c|c|c|c|c|c|c|c|}
\hline \multicolumn{2}{|c|}{ Media } & \multicolumn{2}{|c|}{ Varianza } & \multicolumn{2}{|c|}{ Desviación típica } & \multicolumn{2}{|c|}{$\mathrm{N}$ de elementos } \\
\hline 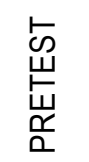 & 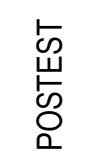 & 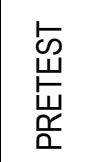 & 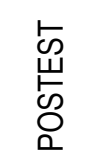 & 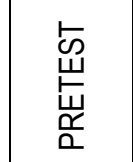 & 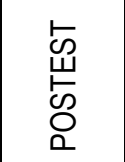 & 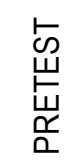 & 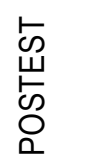 \\
\hline 33.14 & 37.37 & 65.497 & 72.525 & 8.09304 & 8.51619 & 50 & 50 \\
\hline
\end{tabular}


La media en el pretest fue de 33.14 y 37.37 para el postest. Mantiene relación con la media del pilotaje del 38.5, realizado en septiembre del tercer período 2016. La varianza y desviación típica son normales.

Confiabilidad en el pilotaje, el alfa de Cronbach fue de 0.84 para todo el instrumento, el mínimo usualmente aceptado. Según el alfa de Cronbach, la confiabilidad se mantuvo. En el pretest de 0.796 , pero en el postest se recuperó a 0.803 . Asimismo, el postest sin los textos 2 y 5 , que resultó de 0.802 .

Tabla 3. Estadísticos de fiabilidad PRETEST Y POSTEST

\begin{tabular}{|c|c|c|c|c|c|}
\hline \multicolumn{4}{|c|}{ Alfa de Cronbach } & \multicolumn{2}{|c|}{$\mathrm{N}$ de elementos } \\
\hline $\begin{array}{l}\text { 岩 } \\
\stackrel{\circ}{\circ} \\
\bar{\alpha}\end{array}$ & 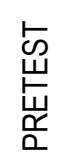 & 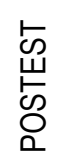 & 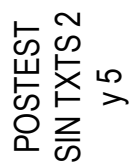 & 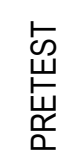 & 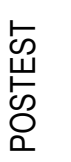 \\
\hline 0.84 & .796 & .803 & .802 & 50 & 50 \\
\hline
\end{tabular}

\section{Análisis de resultados por objetivos de la investigación}

\section{Mejora de la lectura comprensiva}

Comprobar si hay mejora significativa de la destreza de lectura comprensiva en estudiantes de primer ingreso del primer período del 2017 mediante la asignatura de Español General (EG -011), comparando los resultados de un pretest con un postest equivalente que se les aplicará al final de dicho curso.

\section{Resultados Generales}

Al comparar los resultados pretest-postest mediante la diferencia de promedios (V Tabla N.4) se obtuvo una mejoría significativa porcentual de 4.2, o sea se comprueba la hipótesis: Cursar la asignatura de Español General (EG-011) mejora la destreza de lectura comprensiva en la y el estudiante de primer ingreso del 1r período del 2017 en la Ciudad Universitaria de la UNAH.

\section{Resultados por Tipo de Texto}

Los resultados no se esperaban pues en el pilotaje la relación fue inversa (V Tabla .4). El mayor logro se dio en el texto discontinuo "Población Activa" (diagrama de árbol y 
uso público) con $25.8 \%$. La mejora es evidente en la lectura "panorámica" que exige este tipo de diagrama. Se esperaba que en los textos discontinuos tuvieran menor logro, como sucedió en el pilotaje; pero hasta en el texto discontinuo: El Lago Chad (uso educativo y formato de gráfico de curva y de barras) el logro fue de $9.9 \%$, lo que superó al texto narrativo Los Desconocidos (uso literario) con $4.4 \%$, al texto "Noticia del Periódico" (continuo, narrativo- informativo, uso informativo) con $1.8 \%$, y al continuo, expositivo "Emociones Negativas" (uso educativo, tipo cloze) con -2\%: y a Grafi$\mathrm{tti}$ (continuo, argumentativo, uso personal) con -0.8. Por todo esto es que el porcentaje bajó cuando se eliminaron los textos discontinuos de los resultados generales.

El texto Grafitti obtuvo resultado negativo, y esto es inesperado pues en el pilotaje resultó el segundo de mayor puntaje. Se explica por ser el último del primer día, quizás por cansancio. El otro resultado negativo resultó tercero en dificultad en el pretest. Por ser el texto continuo más difícil del segundo día, fue el primero en aplicación, para evitar el cansancio. Aquí el mal resultado no se debe al tipo de texto sino al tipo de lectura, la global, que salió baja como se verá en el siguiente apartado. En estos casos con resultados negativos dicen claramente que en esas secciones los alumnos no dieron su máximo esfuerzo en el postest (o algún factor ambiental), pues la capacidad de lectura comprensiva --como toda destreza mental—no se "olvida" y menos en tres meses. Esto también nos dice que la media de mejora debió ser mayor. El paliativo es que ambos casos no son significativos.

Tabla 4. Porcentaje Promedio de respuestas correctas ajustadas según tipo de texto

\begin{tabular}{|l|l|c|c|}
\hline & Pretest & Post test & Diferencia \\
\cline { 2 - 4 } & Media & Media & Media \\
\hline Porcentaje de respuestas correctas ajustadas" & $33.14 \mathrm{a}$ & $37.37 \mathrm{~b}$ & 4.2 \\
\hline $\begin{array}{l}\text { Porcentaje de respuestas correctas ajustadas Texto 1. "NOTICIA } \\
\text { DE PERIODICO" }\end{array}$ & $42.00 \mathrm{a}$ & $43.80 \mathrm{a}$ & 1.8 \\
\hline $\begin{array}{l}\text { Porcentaje de respuestas correctas ajustadas Texto 2. } \\
\text { "POBLACIÓN ACTIVA" }\end{array}$ & $32.19 \mathrm{a}$ & $57.97 \mathrm{~b}$ & 25.8 \\
\hline $\begin{array}{l}\text { Porcentaje de respuestas correctas ajustadas Texto 3. } \\
\text { "GRAFITTI" }\end{array}$ & $50.06 \mathrm{a}$ & $49.28 \mathrm{a}$ & -.8 \\
\hline $\begin{array}{l}\text { Porcentaje de respuestas correctas ajustadas Texto 4. } \\
\text { "EMOCIONES NEGATIVAS" }\end{array}$ & $24.49 \mathrm{a}$ & $22.52 \mathrm{a}$ & -2.0 \\
\hline $\begin{array}{l}\text { Porcentaje de respuestas correctas ajustadas Texto 5. "EL LAGO } \\
\text { CHAD" }\end{array}$ & $13.96 \mathrm{a}$ & $23.87 \mathrm{~b}$ & 9.9 \\
\hline $\begin{array}{l}\text { Porcentaje de respuestas correctas ajustadas Texto 6. "LOS } \\
\text { DESCONOCIDOS" }\end{array}$ & $18.23 \mathrm{a}$ & $22.62 \mathrm{~b}$ & 4.4 \\
\hline Rapidez Comprensiva & $4.62 \mathrm{a}$ & $5.11 \mathrm{~b}$ & .5 \\
\hline
\end{tabular}




\section{Resultados por Tipo de Lectura.}

Se comprobó la hipótesis con un aumento significativo y progresivo en los tres primeros niveles de lectura (V Tabla5): Estructural, de Primer Nivel y de Segundo Nivel, respectivamente: de 8,9 y 19.5\%. Pero no se comprobó la hipótesis en la lectura global, con $-0.4 \%$. Son buenas noticias el aumento significativo de 25.24 y $44.22=$ $19.5 \%$, en la lectura comprensiva de segundo nivel (intra e interpárrafo), que creció más en relación con las otras dos. La estructural comenzó con menos y creció menos (12.63 y $20.61 \%=8 \%$ ). Es significativo, pero inesperado, por suponerse la lectura más fácil. Es un indicio de que falla la lectura de los signos de puntuación, que delimitan la estructura del texto continuo. La lectura lineal, de comprensión literal de frases y oraciones aisladas, comenzó con más puntos pero no creció mucho en relación con las otras dos (37.54y $46.59=9 \%)$. La lectura global, en cambio, decreció o ganó muy poco. Los datos negativos solo se explican por falta de motivación de estudiante, como ya se dijo, pues como son las preguntas más difíciles no le dieron suficiente esfuerzo en el postest.

\section{Tabla 5. Porcentaje de respuestas correctas ajustadas}

\begin{tabular}{|c|c|c|c|}
\hline & Pretest & Post test & Diferencia \\
\hline & Media & Media & Media \\
\hline Porcentaje de respuestas correctas ajustadas" & $33.14 \mathrm{a}$ & $37.37 \mathrm{~b}$ & 4,2 \\
\hline $\begin{array}{l}\text { Porcentaje de respuestas correctas ajustadas Lectura } \\
\text { estructural" }\end{array}$ & $12.63 a$ & $20.61 b$ & 8,0 \\
\hline $\begin{array}{l}\text { Porcentaje de respuestas correctas ajustadas Lectura } \\
\text { Comprensiva Primer Nivel" }\end{array}$ & $37.54 \mathrm{a}$ & $46.59 b$ & 9,0 \\
\hline $\begin{array}{l}\text { Porcentaje de respuestas correctas ajustadas Lectura } \\
\text { Comprensiva Segundo Nivel" }\end{array}$ & $25.24 a$ & $44.72 b$ & 19,5 \\
\hline $\begin{array}{l}\text { Porcentaje de respuestas correctas ajustadas Lectura } \\
\text { Comprensiva Global" }\end{array}$ & $42.41 \mathrm{a}$ & $35.40 \mathrm{~b}$ & $-7,0$ \\
\hline $\begin{array}{l}\text { Porcentaje de respuestas correctas ajustadas Texto } 4 \text {. } \\
\text { Test CLOZE de lectura global. }\end{array}$ & $24.49 a$ & $22.52 a$ & $-2,0$ \\
\hline $\begin{array}{l}\text { Porcentaje de respuestas correctas ajustadas Texto } 1 \text {. } \\
\text { Test CLOZE de lectura global. }\end{array}$ & $42.00 \mathrm{a}$ & $43.80 \mathrm{a}$ & 1,8 \\
\hline $\begin{array}{l}\text { Porcentaje de respuestas correctas ajustadas Lectura } \\
\text { Comprensiva Global ítems } \mathrm{N}^{\circ} 5 \mathrm{y}\end{array}$ & & & \\
\hline Textos 4 y $1 "$ & $36.90 \mathrm{a}$ & $36.53 a$ &,- 4 \\
\hline $\begin{array}{l}\text { Nota: Los valores de la misma fila y subtabla que } \\
\text { significativamente diferentes en } p<.05 \text { en la prueba } d \\
\text { columnas. Las casillas sin subíndices no se incluyen en la } \\
\text { iguales. } 1 \\
\text { 1. Utilizando la corrección de Bonferroni, se han ajustado la } \\
\text { por pares dentro de una fila para cada subtabla situada má }\end{array}$ & $\begin{array}{l}\text { comp } \\
\text { gual } \\
\text { ueba }\end{array}$ & $\begin{array}{l}\text { mismo } \\
\text { ral de } \\
\text { bas ası }\end{array}$ & $\begin{array}{l}\text { ndice son } \\
\text { as de las } \\
\text { varianzas } \\
\text { araciones }\end{array}$ \\
\hline
\end{tabular}




\section{Correlación del PLEC con la PAA}

Establecer si hay correlación entre los resultados de la prueba de lectura comprensiva aplicada a los estudiantes de primer ingreso (PLEC) y en las pruebas de PAA, como otra forma de evaluar la pertinencia de su programa en función del mejoramiento de esta destreza.Sí hay correlación de todo el PLEC con la PAA (V Tabla 6), pero es media: 0.505 en el pretest y es de 0.523 en el postest. Bajó con respecto al pilotaje, que fue de 0.621 .

Tabla 6. Correlaciones resultados PRETEST y POSTEST PLEC con PAA Verbal Solo casos efectivos

\begin{tabular}{|c|c|c|c|c|c|}
\hline \multirow{2}{*}{} & \multicolumn{2}{|c|}{ PRETEST } & \multicolumn{2}{c|}{ POSTEST } \\
\cline { 3 - 6 } & $\begin{array}{c}\text { \% de RESP } \\
\text { correctas } \\
\text { ajustadas"PLEC" }\end{array}$ & $\begin{array}{c}\text { PAA } \\
\text { Verbal }\end{array}$ & $\begin{array}{c}\text { \% de RESP } \\
\text { correctas } \\
\text { ajustadas"PLEC" }\end{array}$ & $\begin{array}{c}\text { PAA } \\
\text { Verbal }\end{array}$ \\
\hline $\begin{array}{c}\% \text { de } \\
\text { RESP } \\
\text { Correctas } \\
\text { Ajust. PLEC }\end{array}$ & $\begin{array}{c}\text { Correlación } \\
\text { Pearson }\end{array}$ & 1 & $505^{* *}$ & 1 & $523^{* *}$ \\
\cline { 2 - 6 } & $\begin{array}{c}\text { Sig. } \\
\text { (bilateral) }\end{array}$ & & .000 & & .000 \\
\cline { 2 - 6 } & $\mathrm{N}$ & 438 & 431 & 438 & 431 \\
\hline
\end{tabular}

${ }^{* *}$. La correlación es significativa al nivel 0,01 (bilateral).

\section{Reforma Programática}

Determinar si es necesaria una reforma programática de la asignatura de Español General (EG -011) de la UNAH en función de lograr la mejora del nivel de lectura comprensiva, según los resultados obtenidos en esta investigación.

Aunque se prueba la hipótesis, la mejoría obtenida es muy pequeña en todo el PLEC (V Tabla 2): $4.2 \%$ con una media de en 16.5479 en el pretest y de 18.7339 el postest. Los resultados del PLEC sin los textos discontinuos son aún menores: 0.8 y una media de 14.2517. Se evidencia que hay que reformar el programa y mejorar las técnicas didácticas para lograr un mejor aprendizaje en la destreza mental de lectura comprensiva. Ésta es la base de las otras destrezas mentales de comunicación como redacción, la exposición, la disertación, en fin, para estudiar eficientemente.

\section{Datos concretos y fidedignos para orientar la reforma programática}

Obtener datos concretos y fidedignos sobre este problema de bajo nivel de lectura comprensiva y la significatividad de la mejora, si hay, que ayuden a orientar una 
posible reforma programática de la asignatura de Español General (EG-011) de la UNAH en función de lograr mayor competencia en la destreza de lectura comprensiva.

Se correlacionaron 14 variables con los resultados: Edad, Estado conyugal, índice socioeconómico (Análisis factorial), Colegio público o privado, colegio urbano o rural "a" (cabeceras departamentales vrs otros), colegio urbano o rural "b" (Tegucigalpa y SPS vrs otros), Repitió grados en la escuela, Repitió grado en colegio, años que dejó de estudiar después de graduarse de ciclo común, años que dejó de estudiar después de graduarse del nivel preuniversitario, Trabaja actualmente, Horas trabajadas, Horas diaria de Estudio y velocidad de lectura comprensiva.

Los resultados son interesantes y algunos inesperados.

a. Sexo: La mujeres mejoraron 0.64

Edad: el grupo de edad de mayor logro $(10,08)$ es el de $30-34$ años, lo que es inesperado; seguido del grupo 20- 24 con 6.25. (V Tabla 7).

\section{Tabla 7. Resultados por Sexo y edad}

\begin{tabular}{|c|l|l|c|c|c|}
\hline \multicolumn{2}{|l|}{} & Pretest & Post test & Diferencia \\
\hline \multirow{5}{*}{ Sexo } & Potal & PRCA (\% de RESP. correctas ajustadas) & 33,14 & 37,37 & 4,23 \\
\cline { 2 - 6 } & Masculino & PRCA & $33.61 \mathrm{a}$ & $37.38 \mathrm{~b}$ & 3,77 \\
\cline { 2 - 6 } & Femenino & PRCA & $32.86 \mathrm{a}$ & $37.37 \mathrm{~b}$ & 4,51 \\
\hline Edad & $15-19$ & PRCA (\% de RESP. correctas ajustadas) & $34.56 \mathrm{a}$ & $38.45 \mathrm{~b}$ & 3,88 \\
\cline { 2 - 6 } & $20-24$ & PRCA & $26.37 \mathrm{a}$ & $32.62 \mathrm{~b}$ & 6,25 \\
\cline { 2 - 6 } & $25-29$ & PRCA & $32.27 \mathrm{a}$ & $31.90 \mathrm{a}$ &,- 37 \\
\cline { 2 - 6 } & $30-34$ & PRCA & $28.25 \mathrm{a}$ & $38.33 \mathrm{a}$ & 10,08 \\
\cline { 2 - 6 } & 35 o más & PRCA & $26.38 \mathrm{a}$ & $34.13 \mathrm{a}$ & 7,75 \\
\hline
\end{tabular}

Estado conyugal: También inesperado ( $\mathrm{V}$ Tabla 8): los casados tienen el mayor logro (5.54), seguido de los divorciados (5.23), ambos iniciaron con menos puntaje que los solteros, quienes aumentaron menos.

Índice socioeconómico (Análisis factorial): Aquí también hay inesperados (V Tabla N.9): el grupo del tercer cuartil logró más (6.17) seguido del que tiene peor situación económica, con menos haberes, en lugar del grupo con más haberes 
Tabla 8. Resultados por estado conyugal

\begin{tabular}{|c|c|c|c|c|c|}
\hline \multirow{4}{*}{$\begin{array}{c}\text { Estado } \\
\text { conyugal }\end{array}$} & $\begin{array}{c}\text { PRCA (\% de RESP } \\
\text { correctas ajustadas) }\end{array}$ & $33.42 \mathrm{a}$ & $37.23 \mathrm{a}$ & 3,81 \\
\cline { 2 - 6 } & Soltero(a) & PRCA & $33.71 \mathrm{a}$ & $37.87 \mathrm{~b}$ & 4,16 \\
\cline { 2 - 6 } & Casado (a) o Unido (a) & PRCA & $26.96 \mathrm{a}$ & $32.50 \mathrm{a}$ & 5,54 \\
\cline { 2 - 6 } & $\begin{array}{c}\text { Otro: Separado(a)/ } \\
\text { Divorciado(a)/Viudo(a }\end{array}$ & PRCA & $27.68 \mathrm{a}$ & $32.91 \mathrm{a}$ & 5,23 \\
\hline
\end{tabular}

$a=$ significativo

$b=$ no significativo

Tabla 9. Resultados por Índice socioeconómico (Análisis factorial)

\begin{tabular}{|c|c|c|c|c|c|}
\hline \multirow{2}{*}{$\begin{array}{c}\text { Índice } \\
\text { socioeconómico } \\
\text { (Análisis } \\
\text { factorial) }\end{array}$} & Cuartil 1 & $\begin{array}{c}\text { PRCA \% de RESP } \\
\text { correctas ajustadas }\end{array}$ & $31.03 \mathrm{a}$ & $36.39 \mathrm{~b}$ & 5,35 \\
\cline { 2 - 6 } & Cuartil 2 & PRCA & $31.44 \mathrm{a}$ & $34.78 \mathrm{a}$ & 3,34 \\
\cline { 2 - 6 } & Cuartil 3 & PRCA & $34.29 \mathrm{a}$ & $40.45 \mathrm{~b}$ & 6,17 \\
\cline { 2 - 6 } & Cuartil 4 & PRCA & $35.71 \mathrm{a}$ & $37.90 \mathrm{a}$ & 2,19 \\
\hline
\end{tabular}

$a=$ significativo $\quad b=$ no significativo

Colegio público o privado. También aquí se observa algo nuevo (V Tabla 10), pues el grupo del colegio público, obtuvo mayor logro (1.39), pero comenzó con menor puntaje que el de colegios privados.

Tabla 10. Resultados por administración privada o pública del colegio de egreso

\begin{tabular}{|c|c|c|c|c|c|}
\hline \multirow{2}{*}{ Administra } & Público & $\begin{array}{c}\text { PRCA (\% de RESP } \\
\text { correctas ajustadas) }\end{array}$ & $31.23 \mathrm{a}$ & $35.97 \mathrm{~b}$ & 4,74 \\
\cline { 2 - 6 } & Privado & PRCA & $36.24 \mathrm{a}$ & $39.59 \mathrm{a}$ & 3,35 \\
\hline
\end{tabular}

$a=$ significativo $\quad b=$ no significativo

Colegio urbano o rural "a" (cabeceras departamentales vrs. otros) y "b" Colegio urbano o rural (Tegucigalpa y SPS vrs otros): Se confirma (V Tabla 11) que las cabeceras departamentales obtienen mayor logro que los pueblos: $3.85 \%$. Igual, se confirma que Tegucigalpa y San Pedro Sula obtienen mayor logro que los demás: $2.75 \%$; sin embargo, entraron con menos puntaje. Esto es inesperado.

Tabla 11. Resultados por lugar urbano o rural del colegio de egreso

\begin{tabular}{|c|c|c|c|c|c|}
\hline $\begin{array}{c}\text { Tipo de lugar donde } \\
\text { está el colegio }\end{array}$ & $\begin{array}{c}\text { Cabec. } \\
\text { Deptal }\end{array}$ & $\begin{array}{c}\text { PRCA (\% de RESP } \\
\text { correctas ajustadas) }\end{array}$ & $33.29 \mathrm{a}$ & $38.05 \mathrm{~b}$ & 4,76 \\
\cline { 2 - 6 } & Pueblo & PRCA & $32.66 \mathrm{a}$ & $34.57^{\mathrm{a}}$ & 1,91 \\
\hline $\begin{array}{c}\text { Tipo de lugar donde } \\
\text { está el colegio }\end{array}$ & Teg/SPS & PRCA & $32.70 \mathrm{a}$ & $37.66 \mathrm{~b}$ & 4,96 \\
\cline { 2 - 7 } & Otros & PRCA & $34.52 \mathrm{a}$ & $36.72^{\mathrm{a}}$ & 2,21 \\
\hline
\end{tabular}

$a=$ significativo $\quad b=$ no significativo 
Repitencia (V Tabla 11): Repitió grados en la escuela: También inesperado que los alumnos alguna vez repitentes tienen mayor logro: $2.08 \%$ a pesar de haber comenzado en desventaja.

Repitió grado en colegio ( $\mathrm{V}$ Tabla 11). También inesperado que los alumnos alguna vez repitentes tienen mayor logro: $2.45 \%$, a pesar de haber comenzado en desventaja.

Tabla 12. Resultados por repitencia de grados en la escuela y el colegio

\begin{tabular}{|c|c|c|c|c|c|}
\hline $\begin{array}{c}\text { Repitió } \\
\text { grados en la } \\
\text { escuela }\end{array}$ & Sí & PRCA (\% de RESP correctas ajustadas) & $24.83 \mathrm{a}$ & $31.02 \mathrm{a}$ & 6,19 \\
\hline \multirow{2}{*}{$\begin{array}{c}\text { Repitió grado } \\
\text { en colegio }\end{array}$} & Sí & PRCA (\% de RESP correctas ajustadas) & $24.77 \mathrm{a}$ & $31.39 \mathrm{a}$ & 6,62 \\
\cline { 2 - 6 } & No & PRCA & $33.92 \mathrm{a}$ & $37.89 \mathrm{~b}$ & 3,97 \\
\hline
\end{tabular}

$a=$ significativo $\quad b=$ no significativo

Años de demora en ingresar al siguiente nivel educativo (V Tabla 13)

Años que dejó de estudiar después de graduarse de ciclo común: También inesperado que los alguna vez demorados tienen mayor logro: $1.51 \%$, a pesar de haber comenzado en desventaja.

Años que dejó de estudiar después de graduarse de diversificado: También inesperado que los alguna vez demorados tienen mayor logro: $4.73 \%$, a pesar de haber comenzado con una desventaja de casi 7 puntos. Interesante también que el grupo que no se demoró no obtuvo ni un punto de logro, a pesar de haber comenzado con esa ventaja.

Tabla 13. Resultados por demora en ingresar al siguiente nivel educativo

\begin{tabular}{|c|l|c|c|c|c|}
\hline $\begin{array}{c}\text { Dejo de estudiar } \\
\text { después de } \\
\text { graduarse de ciclo } \\
\text { común }\end{array}$ & Sí & $\begin{array}{c}\text { PRCA (\% de RESP } \\
\text { correctas ajustadas) }\end{array}$ & $29.81 \mathrm{a}$ & $35.44 \mathrm{a}$ & 5,64 \\
\cline { 2 - 6 } & No & PRCA & $33.33 \mathrm{a}$ & $37.46 \mathrm{~b}$ & 4,13 \\
\hline $\begin{array}{c}\text { Dejo de estudiar } \\
\text { después de } \\
\text { graduarse de } \\
\text { diversificado }\end{array}$ & Sí & $\begin{array}{c}\text { PRCA (\% de RESP } \\
\text { correctas ajustadas) }\end{array}$ & $29.02 \mathrm{a}$ & $34.41 \mathrm{a}$ & 5.39 \\
\cline { 2 - 6 } & No & PRCA & $35.93 \mathrm{a}$ & $36.59 \mathrm{a}$ & .66 \\
\hline
\end{tabular}

$a=$ significativo $\quad b=$ no significativo 
Horas de trabajo (V Tabla 14)

Trabaja actualmente: El grupo que trabaja logró $0.84 \%$ sobre el que no trabaja.

Horas trabajadas: el grupo de mayor logro $(9,22)$ es el que trabaja 7 horas, lo que es inesperado; seguido del grupo con 3 horas: con 8.50 .

\section{Tabla 14. Resultados por trabajar o no y horas trabajadas}

\begin{tabular}{|l|c|c|c|c|c|}
\hline $\begin{array}{l}\text { Trabaja } \\
\text { actualmente }\end{array}$ & Sí & $\begin{array}{c}\text { PRCA (\% de RESP correctas } \\
\text { ajustadas) }\end{array}$ & $33.05 \mathrm{a}$ & $37.93 \mathrm{a}$ & 4,88 \\
\cline { 2 - 6 } & No & PRCA & $33.24 \mathrm{a}$ & $37.27 \mathrm{~b}$ & 4,04 \\
\hline $\begin{array}{l}\text { Horas } \\
\text { trabajadas }\end{array}$ & 3 & $\begin{array}{c}\text { PRCA (\% de RESP correctas } \\
\text { ajustadas) }\end{array}$ & 36.501 & 45.001 & 8,50 \\
\cline { 2 - 6 } & 4 & PRCA & $49.00 \mathrm{a}$ & $45.50 \mathrm{a}$ & $-3,50$ \\
\cline { 2 - 6 } & 5 & PRCA & $30.19 \mathrm{a}$ & $31.94 \mathrm{a}$ & 1,75 \\
\cline { 2 - 6 } & 6 & PRCA & $40.80 \mathrm{a}$ & $45.40 \mathrm{a}$ & 4,60 \\
\cline { 2 - 6 } & 7 & PRCA & $28.61 \mathrm{a}$ & $37.83 \mathrm{a}$ & 9,22 \\
\cline { 2 - 6 } & 8 o más & PRCA & $32.55 \mathrm{a}$ & $37.71 \mathrm{a}$ & 5,16 \\
\cline { 2 - 6 } & No trabaja & PRCA & $33.24 \mathrm{a}$ & $37.27 \mathrm{~b}$ & 4,04 \\
\hline
\end{tabular}

$$
a=\text { significativo } \quad b=\text { no significativo }
$$

Horas diarias de Estudio (V Tabla 15), extrañamente el grupo que tiene 5 horas de estudio tuvo más logros (7.49), y el de seis o más horas, mucho menos logro (2.29). El siguiente mayor logro es del grupo que estudia dos horas, con $7.49 \%$.

\section{Tabla 15. Resultados por horas de estudio}

\begin{tabular}{|c|c|c|c|c|c|}
\hline $\begin{array}{c}\text { Horas de } \\
\text { Estudio }\end{array}$ & .00 & $\begin{array}{c}\text { PRCA (\% de RESP correctas } \\
\text { ajustadas) }\end{array}$ & $47.23 \mathrm{a}$ & $47.77 \mathrm{a}$ &, 55 \\
\hline \multirow{6}{*}{} & 1.00 & PRCA & $32.20 \mathrm{a}$ & $36.55 \mathrm{a}$ & 4,35 \\
\cline { 2 - 6 } & 2.00 & PRCA & $32.77 \mathrm{a}$ & $40.26 \mathrm{~b}$ & 7,49 \\
\cline { 2 - 6 } & 3.00 & PRCA & $34.88 \mathrm{a}$ & $37.15 \mathrm{a}$ & 2,27 \\
\cline { 2 - 6 } & 4.00 & PRCA & $31.73 \mathrm{a}$ & $33.76 \mathrm{a}$ & 2,02 \\
\cline { 2 - 6 } & 5.00 & PRCA & $31.23 \mathrm{a}$ & $36.37 \mathrm{a}$ & 5,14 \\
\cline { 2 - 6 } & 6 o más & PRCA & $32.15 \mathrm{a}$ & $34.44 \mathrm{a}$ & 2,29 \\
\hline
\end{tabular}

Nota: Los valores de la misma fila y subtabla que no comparten el mismo subíndice son significativamente diferentes en $p<.05$ en la prueba de igualdad bilateral de medias de las columnas. Las casillas sin subíndices no se incluyen en la prueba. Las pruebas asumen varianzas iguales. 2 
Esta categoría no se utiliza en las comparaciones porque la suma de ponderaciones de los casos es inferior a dos.

No se realizan comparaciones por pares para algunas subtablas debido a problemas numéricos.

Rapidez comprensiva: Sólo aumentó 0.5 .

Procedimientos Docentes que ayudan a mejorar la lectura comprensiva. Identificar procedimientos docentes que ayudan a mejorar la destreza de lectura comprensiva para procurar su generalización, mediante entrevista a los catedráticos cuyas secciones obtengan mayores puntajes en los resultados del pretest-postest.

En casi todas las secciones investigadas se obtuvo mejoría parecida y baja (V Tabla 16), a excepción de la sección 1204 con 14.31\%. Con esta "máxima" nota de 14.31\%, se puede decir que de 10.6 a 14 es el cuartil "superior", de 7 a 10.5 es el medio "superior"; de 3.5 a 7 es el cuartil "medio inferior", y así el primer cuartil. Con esta escala, una sección está en el cuartil superior, y cinco en el medio superior. Éstas fueron las seleccionadas para entrevistar los docentes. Los datos a continuación:

Tabla 16. Resultados por secciones

\begin{tabular}{|l|l|c|c|c|}
\hline \multicolumn{2}{|l|}{ Sección } & Pretest & Post test & Diferencia \\
\hline Total & PRCA & 33,14 & 37,37 & 4,23 \\
\hline 600.00 & PRCA & $42.10 \mathrm{a}$ & $50.31 \mathrm{a}$ & 8,21 \\
\hline 701.00 & PRCA & $29.07 \mathrm{a}$ & $38.10 \mathrm{a}$ & 9,03 \\
\hline 803.00 & PRCA & $43.08 \mathrm{a}$ & $37.25 \mathrm{a}$ & $-5,83$ \\
\hline 903.00 & PRCA & $39.50 \mathrm{a}$ & $38.24 \mathrm{a}$ & $-1,26$ \\
\hline 1001.00 & PRCA & $26.35 \mathrm{a}$ & $31.81 \mathrm{a}$ & 5,45 \\
\hline 1102.00 & PRCA & $45.13 \mathrm{a}$ & $42.60 \mathrm{a}$ & $-2,53$ \\
\hline 1204.00 & PRCA & $27.65 \mathrm{a}$ & $41.96 \mathrm{~b}$ & 14,31 \\
\hline 1301.00 & PRCA & $23.98 \mathrm{a}$ & $33.78 \mathrm{~b}$ & 9,80 \\
\hline 1401.00 & PRCA & $37.84 \mathrm{a}$ & $37.00 \mathrm{a}$ &,- 84 \\
\hline 1501.00 & PRCA & $34.73 \mathrm{a}$ & $36.34 \mathrm{a}$ & 1,61 \\
\hline 1502.00 & PRCA & $28.82 \mathrm{a}$ & $32.97 \mathrm{a}$ & 4,15 \\
\hline 1600.00 & PRCA & $34.64 \mathrm{a}$ & $31.80 \mathrm{a}$ & $-2,84$ \\
\hline 1701.00 & PRCA & $33.13 \mathrm{a}$ & $35.27 \mathrm{a}$ & 2,13 \\
\hline 1800.00 & PRCA & $30.23 \mathrm{a}$ & $36.96 \mathrm{a}$ & 6,73 \\
\hline 1900.00 & PRCA & $28.05 \mathrm{a}$ & $36.95 \mathrm{~b}$ & 8,90 \\
\hline 2000.00 & PRCA & $34.46^{*}$ & $42.10^{*}$ & 7,64 \\
\hline \multicolumn{5}{|c|}{$\mathrm{b}=$ no significativo } \\
\hline
\end{tabular}


Esto resultados no son contundentes porque en los datos por sección se denotan más los factores intervinientes, como el tipo de grupo (en general: bueno, regular, malo), la situación ambiental y motivacional tanto de alumnos como docentes, los procedimientos didácticos usados, las interferencias específicas que hayan sucedido en esa clase específica, la cantidad de pruebas válidas aplicadas y su proporción en la muestra, etc. Esto también incide en la significatividad de los datos de cada sección.

En los casos negativos se denota la poca motivación o actitud no interesada del grupo (o algún factor ambiental coyuntural) que no dio su máximo esfuerzo en el postest, pues las destrezas, máxime las mentales como la capacidad en lectura comprensiva, no se olvidan y menos en tres meses. Además, sin duda durante ese tiempo se practicó la lectura en la clase de Español General (EG-011) y en las demás clases que llevara el estudiante. Esto también nos dice que la mejora debió ser mayor.

Llama la atención que los seis mejores logros están en los "extremos": 6 y 7 a.m., 12 y 1 p.m. y 7 y 8 p.m.

\section{DISCUSIÓN}

Aunque se prueba la hipótesis, la mejoría obtenida es muy pequeña en todo el PLEC: $4.2 \%$. Por tanto, debe reformarse el programa de la asignatura de Español General (EG-011). Los resultados por secciones no son contundentes porque aquí se denotan más los factores intervinientes. En los casos negativos se denota la poco motivación 0 actitud no interesada del grupo (o algún factor ambiental coyuntural) que no dio su máximo esfuerzo en el postest. Esto también nos dice que la media de mejora debió ser mayor. Debe buscarse un mecanismo para lograr que los alumnos le presten interés suficiente a este tipo de evaluaciones. Es necesario corroborar con un estudio similar, si todos estos resultados son tendencia o coyuntura.

\section{CONCLUSIONES}

1. Resultados por nivel de lectura. Se comprobó hay mejora significativa de la capacidad de lectura comprensiva en el estudiantado que cursó la asignatura de Español 
General en el primer período 2017 con $4.23 \%$ de logro general, en los tres primeros niveles de lectura (Estructural: 8; Primer Nivel: 9; Segundo Nivel: 19.5\%. Pero la lectura global decreció $(-0.4 \%)$. El segundo nivel creció más en relación que las otros dos, lo cual es un logro importante. La estructural comenzó con menos y creció menos (12.63 y $20.61 \%=8 \%$ ). Es significativo, pero es inesperado, puede suponerse que falla la lectura de los signos de puntuación. La lectura lineal comenzó con más puntos pero no creció mucho en relación con las otras dos (37.54 y $46.59=9 \%)$.

2. Resultados por tipo de texto. El texto discontinuo de diagrama de árbol y uso público, logró el mayor puntaje de todos con $25.8 \%$, evidenciando mejora en la lectura "panorámica" que exige este tipo de diagrama. En el texto discontinuo de uso educativo y formato de gráfico de curva y de barras, el logro fue de 9.9\%. Lo inesperado es que superó al texto continuo narrativo (uso literario) con $4.4 \%$, al texto continuo, narrativo-informativo, uso informativo, con 1.8\%, y al continuo, expositivo, uso educativo, tipo cloze con $-2 \%$ : y al texto continuo, argumentativo, uso personal con -0.8. Por todo esto es que el porcentaje bajó cuando se eliminaron estos textos de los resultados generales. Estos resultados no se esperaban pues en el pilotaje la relación fue inversa.

El texto continuo, argumentativo, uso personal (Grafitti), resultó negativo, inesperadamente, pues en el pilotaje resultó el segundo mayor. Se explica por ser el último del primer día, en que se denota la poca motivación del estudiantado por contestarlo, quizás por cansancio. El otro resultado negativo resultó tercero en dificultad en el pilotaje. Por ser el texto continuo más difícil del segundo día, fue el primero en aplicación, para evitar el cansancio. Aquí el mal resultado no se debe al tipo de texto sino al tipo de lectura, la global, que salió baja como se verá en el siguiente apartado. En estos casos con resultados negativos se denota la actitud no interesada del grupo (o algún factor ambiental), pues la capacidad de lectura comprensiva --como toda destreza mental ya adquirida—no se olvida, y menos en tres meses.. Esto también nos dice que la media de mejora debió ser mayor. El paliativo es que ambos casos no son significativos. Debe buscarse un mecanismo para lograr que los alumnos le presten interés suficiente a este tipo de evaluaciones. Por ejemplo, se propone que su resultado final incida en la nota del curso, quizás como puntos extras.

3. Hay correlación media de todo el PLEC con la PAA: 0.505 en el pretest y es de 0.523 en el postest. Bajó con respecto al pilotaje, que fue de 0.621 . 
4. Hay que reformar el programa de Español General y mejorar las técnicas didácticas para lograr un mejor aprendizaje en las destrezas mentales de lectura comprensiva, ya que el porcentaje de logro es muy bajo: 4.23 , al igual que la media: 33.14 en el pretest y 37.37 en el postest.

5. Las 14 variables de la encuesta que se correlacionaron con los resultados finales proporcionan una buena descripción de la situación y fenómenos estudiado, lo que sirve de importante base para una reforma del programa de Español General, y para otras clases y decisiones políticos-académicas de la UNAH. Los resultados son interesantes y algunos inesperados.

6. En todas las secciones investigadas se obtuvo muy bajo logro, el mejor fue la sección 1204 con 14.31\%. Con esta "máxima" nota se establecieron cuartiles "superior" (10.6 a 14), "medio superior", de 7 a 10.5; "medio inferior" de 3.5 a 7 ; e inferior. Así, hay un caso en el cuartil "superior" y cinco en el "medio superior"

Llama la atención que los seis mejores logros están en los "extremos": 6 y 7 a.m., 12 y 1 p.m. y 7 y 8 p.m. Estos resultados no son contundentes porque en los datos por sección se denotan más los factores intervinientes. Esto también incide en la significatividad de los datos de cada sección.

Los docentes cuyas secciones obtuvieron los mejores seis logros tienen en común: las lecturas que asignan son evaluadas con ensayo corto o preguntas expositivas o de respuesta breve, pero de interpretación del texto en el mismo o en el contexto social. Además, cinco usan varias fuentes como texto teórico, y todos evalúan con items de aplicación, no solo de memoria. Hacen además actividades que se salen de la clase tradicional: una investigación grupal sobre un autor hondureño y los jueves el docente les ayuda en la investigación y la redacción que presentan en la tercera unidad; una lectura de poesía individual en la entrada de la UNAH, un cortometraje grupal con sus celulares sobre alguna narración y para esto, les enseña a hacer guiones; hacen sociodramas, ver teatro y cine como textos audiovisuales e interpretarlos; elaborar un libro con tema asignado y dirigirlos en clase durante todo el proceso.

7. Es necesario darle continuidad a esta investigación, para corroborar si los resultados actuales son tendenciales o coyunturales y darle seguimiento a las preguntas que dio, como buen estudio descriptivo.

8. Es deseable aplicar esta prueba a todas las secciones de Español General y optati- 
vas, para detectar dificultades y logros en la programación, docencia y en la institución. Pruebas similares deberían desarrollarse y aplicarse en las otras asignaturas, al menos las generales, pues las competencias en destrezas mentales son herramientas que no solo se necesitan en la asignatura de español, sino en todos los estudios y en la práctica profesional.

9. Es posible y deseable continuar esta investigación de forma longitudinal: dentro de unos tres años, 0 al final de sus estudios, localizar a los estudiantes a quienes se les aplicó este instrumento, y volvérselos a aplicar, para conocer cuánto han mejorado con respecto a su último logro, y así saber cuánto incide la universidad en el mismo

10. La PLEC superó la prueba del pilotaje. Pero no se esperaba tanta informalidad de varios estudiantes, que no hicieron el segundo día de ambas etapas o todo el postest (en este caso, algunos ya no cursaban la clase, lo que sí se previó). En adelante, su aplicará un instrumento más corto, para evitar la pérdida de informantes. Quedarán entonces los textos 2, 3, 4, 6 y 7 (de rapidez comprensiva, que dura un minuto). Al eliminar pruebas, la confiabilidad baja. Por eso se debe aumentar la cantidad de ítems, siempre cuidando de que correspondan a los cuatro niveles de lectura trabajados en esta investigación: 8010 ítems por texto. Se considerará otro texto literario narrativo más simple, para corroborar si el bajo logro en éste se debe a la puntuación más literaria del mismo, supuesto que es coherente con el bajo logro en la lectura estructural. En este caso, se necesitará probablemente 50 minutos para aplicar la PLEC. La encuesta socioeconómica se aplicará al día siguiente. O sea que la aplicación de la PLEC insumirá hora y media en el pretest y una hora en postest.

\section{AGRADECIMIENTOS}

A los licenciados: Sue Laínez, Carlos Obed Velásquez, Daysi Velázquez, Marcela Carías, Digna Hernández, Yanira Durán, Sergio Rivera, Johana Burgos, Lorena Suazo, Nelson Ordóñez, se les agradece todo su apoyo y esfuerzo al colaborar con este proyecto como revisores de la prueba piloto, pues éste es un trabajo extra no remunerado y todos tenían entre manos mucho trabajo propio académico y personal. Igualmente a los licenciados Sergio Rivera, Fanny Meléndez, Lorena Suazo, Maira Cruz, Carmen Velásquez, Oscar Amaya, Luis David Reyes, Kaby Burgos, Yanira Durán, Digna Hernández, Martha Rodríguez, Dilcia Osorto, por permitir realizar esta 
investigación en su clase de Español General (EG-011). También a la doctora Socorro Castellón que me guió en la teoría y aplicación de los instrumentos y al máster Ramón Enamorado quien ejecutó en todo el trabajo de programación y manejo estadístico de la gran cantidad de datos recabados. Agradecemos aquí la muy pronta colaboración que nos dio la Dirección de Admisión, al cargo de la lic. Rita Tamashiro y su equipo, al solicitarles los resultados de las PAA de los estudiantes que hicieron nuestro pilotaje y PLEC, para correlacionar ambas pruebas. También a Saris Elena Díaz y César Fernando Ponce, quienes me apoyaron como secretarios en muchas ocasiones, pues este trabajo es demasiado para una sola persona. Y claro, se agradece a la DICYP-UNAH pues sin la beca otorgada no habría sido posible esta investigación.

\section{REFERENCIAS BIBLIOGRÁFICAS}

Espinoza-Ocaña, L., \& Orantes-García, C. (Junio de 2014). Viabilidad y germinación de Guaiacum sanctum L. (Zygophyllaceae), árbol tropical amenazado. LACANDONIA, 8(1), 37-40.

Ferrufino, L., López, T., Suazo, L., \& Díaz, R. (25 de Mayo de 2017). La colección de especies nativas del bosque seco tropical de Honduras: un laboratorio de campo. Centro de Investigación Científica de Yucatán, 93-97.

Ferrufino-Acosta, L., Mejía-Ordóñez, T. M., \& Corrales-Andino, R. E. (Diciembre de 2016). Estudio Poblacional de Guaiacum sanctum L. (Zygophyllaceae) en los bosques secos de Honduras. Revista Ciencia y Tecnología(19), 78-93.

Fuchs, E., \& Hamrick, J. (2010). Genetic Diversity in the Endagered Tropical Tree Guaiacum sanctum (Zygophyllaceae). Journal of Heredity, 101(3), 284-291.

Hernández, M. I., Lobo , M., Medina, C. I., Régulo-Cartagena, J., \& Delgado, O. A. (2009). Comportamiento de la germinación y categorización de la latencia en semillas de mortiño (Vaccinium meridionale Swartz). Agronomía Colombiana, 27, 15-23.

Ríos de León, N. d. (Noviembre de 2016). Germinación in vitro de Guaiacum sanctum L. (Zygophyllaceae) como alternativa de conservación y aprovechamiento sustentable. Universidad de Ciencias y Artes de Chiapas. Instituto de Ciencias Biológicas , 1-95.

Rivers, M. (2017). Guaiacum sanctum. The IUCN Red List of Threatened Species 2017. Obtenido de http://dx.doi.org/10.2305/IUCN.UK.2017-3.RLTS.T32955A68085952.en

Sánchez, D. (Octubre de 1995). Determinación del potencial germinativo de tres especies maderables nativas de un bosque húmedo tropical de Nicaragua. Avances en la producción de semillas forestales en Ámerica Latina, 203-206.

Sinha. (2014). Modern Plant Physiology. Oxford: Alpha Science Internantional.

Taylor, A. (1966). Seed germination and seedling growth of Guaiacum sanctum L. Florida State Horticultural Society, 79, 468-470. 
Varela, S., \& Arana, V. (2011). Latencia y Germinanción de Semillas. Tratamientos Pregerminativos. Serie ténica: Sistemas Forestales Integrados, 1-10.

Victoria, J., Bonilla, C., \& Sánchez, M. (2006). Viabilidad en tetrazolio de semillas de caléndula y eneldo. Acta Agronómica, 55(1), 1-15. 